\section{Adverse drug events in hospitals: a systematic review}

\author{
Eventos adversos a medicamentos em hospitais: \\ uma revisão sistemática
}

\author{
1 Universidade Estadual do \\ Oeste do Paraná, Cascavel, \\ Brasil. \\ 2 Escola Nacional de Saúde \\ Pública Sergio Arouca, \\ Fundação Oswaldo Cruz, \\ Rio de Janeiro, Brasil. \\ Correspondence \\ F. G. Cano \\ Universidade Estadual do \\ Oeste do Paraná. \\ Rua Universitária 1619, \\ Cascavel, $P R$ \\ 85819-110, Brasil \\ fgiordanicano@yahoo.com.br
}

\begin{abstract}
The objective of this study was to evaluate studies on the occurrence of adverse drug events (ADEs) in hospitals in order to learn about their frequency and characteristics, comparing the methods for identifying them and the various definitions. A search was conducted on MEDLINE and identified studies published from 2000 to 2009. Inclusion criteria were: studies in populations not selected for specific diseases or drugs and ADEs that occurred during hospitalization. Twenty-nine studies were selected, displaying multiple sources of heterogeneity, including differences in the study populations, surveillance techniques, definitions of ADEs, and indicators. The proportion of patients with ADEs ranged from $1.6 \%$ to $41.4 \%$ of inpatients and the rates ranged from 1.7 to 51.8 events 100 admissions. A considerable share of these events could have been avoided. The findings show that ADEs in inpatients are a public health problem. However, further studies are needed to monitor these adverse events in order to effectively promote safe drug use.
\end{abstract}

Drug Monitoring; Drug Therapy; Pharmacoepidemiology
Fabíola Giordani Cano 1

Suely Rozenfeld 2

\section{Introduction}

Drugs currently represent an important therapeutic strategy and are widely used, especially in the hospital setting. However, there are inherent risks in their pharmacological action or related to their use, which can lead to the development of adverse drug events (ADEs), otherwise known as adverse drug reactions (ADRs).

In order to guarantee the safe use of medicines, it is necessary to monitor the occurrence of post-registration/post-marketing ADEs, a process known as pharmacovigilance. This strategy aims to assist regulatory activities in the patient safety area.

A pioneering initiative in monitoring adverse drug reactions in inpatients was the Boston Collaborative Drug Surveillance Program (BDSCP), launched in 1966, which conducted an active search for events and collected data on 35,000 patients in ten years 1 . In another study, Seidl et al. 2 conducted an active search for events and estimated their frequency at $13.6 \%$ among inpatients. Since then, numerous studies have been published, although with widely varying estimates of frequency.

In 1998, a meta-analysis estimated the incidence of severe ADRs at $6.7 \%(5.2 \%-8.2 \%) 3$, but this finding should be interpreted with caution due to various sources of heterogeneity among the studies 4 . 
The early 1990s witnessed a new stage in the issue of drug safety, which in addition to recognizing the production of adverse events, even when drugs are used appropriately, also identifies the possibility that drugs can cause harmful effects due to flaws or errors during the complex process of their clinical utilization 5 .

In this context, the aim of the current review was to evaluate studies on the occurrence of ADEs in the hospital setting in order to determine their frequency and characteristics, comparing the techniques for their identification and the concepts used to describe them.

\section{Method}

This was a systematic literature review with a search for articles published from 2000 to August 2009.

The electronic search strategy included the MEDLINE database, using the PubMed interface. The search equation consisted of the descriptors "adverse drug reaction reporting systems", "drug therapy/adverse effects", and "pharmaceutical preparations/adverse effects" to retrieve studies on adverse events. Added to these were either the descriptors "hospitals", "hospitals, veterans", "hospitalization”, "inpatients", "pharmacy service, hospital", "hospital units", and "medication systems, hospital" or the terms "hospital*" and “inpatient" in the title and abstract. The limits on the search strategy were the period of publication (January 1, 2000, to August 24, 2009), type of population (humans), and type of study (excluding editorials, letters, reviews, and cases). We also excluded articles whose titles included the terms "child", "children", "pediatric", or "emergency".

The articles retrieved through the electronic search were submitted to exclusion criteria in order to select those that captured the variety of events occurring in patients admitted to general hospitals. This selection process followed the stages described below.

First, the articles' titles were evaluated independently by the authors, considering the exclusion of studies focusing on: events associated with specific drugs, organs, or systems, and events occurring in hospitals or clinics involving clinical specialties, outpatient services, intensive care units, emergency departments, community care, or nursing home. We also excluded studies that only evaluated ADEs that led to hospitalization or that occurred after discharge, plus those that only approached elderly or pediatric populations.

Next, the same exclusion criteria were applied to the article abstracts, also excluding those that only approached serious events, those that produced sequelae or were fatal, or exclusively focused on medication errors. We did not include studies that specifically approached the utilization of medicines and pharmaceutical care, health professionals' or patients' opinions, or presentation of programs to improve ADE detection systems, since they failed to provide relevant data for our purposes. We only included full texts available in English, Portuguese, Spanish, or French. Occasional disagreements between the two reviewers were resolved by discussion until reaching a consensus.

The complete texts of the remaining articles (plus those that lacked abstracts) were read in full. During this stage, exclusions were performed according to the previously mentioned criteria or when the articles presented prevalence data on sub-samples of studies already included in the review. Articles that only identified events by means of spontaneous reporting were excluded, because the latter method underreports the events and thus hinders comparison with other studies.

The same inclusion and exclusion criteria were used to select the studies retrieved by manual search in the bibliographic references of the selected articles. The search strategy was also performed in the LILACS database, but detected no additional articles for inclusion.

The expression "adverse drug event" is used throughout the text to characterize harm caused by the use of medicines.

Data collection used a standardized form that was pretested with three data. Article extraction was performed by F.G.C. and reviewed by S.R.

The EndNote software (Thomson Reuters; http://www.endnote.com) was used to organize the bibliographic references.

\section{Results}

The search strategy retrieved 1,817 articles, and Figure 1 shows the selection stages with the results of the application of the inclusion and exclusion criteria. Twenty-eight articles remained $6,7,8,9,10,11,12,13,14,15,16,17,18,19,20,21,22,23,24,25,26,27,28$, 29,30,31,32,33 for evaluation, plus one more article retrieved by manual search 34 .

Table 1 describes the studies, conducted in 13 countries from North America, Europe, South America, andAsia.Ten studies 8,9,15,16,18,19,22,23,24,28 $(34.5 \%)$ were conducted in the United States and two $(6.9 \%)$ in Brazil 20,29.

Most of the studies were performed in one hospital (19/29) 7,8,12,13,15,16,17,18,19,20,21,25,26,27,28, $31,32,33,34$. Six were multi-center 6,9,22,23,24,29 and 
Flowchart of systematic article search and selection on in-hospital adverse drug events.

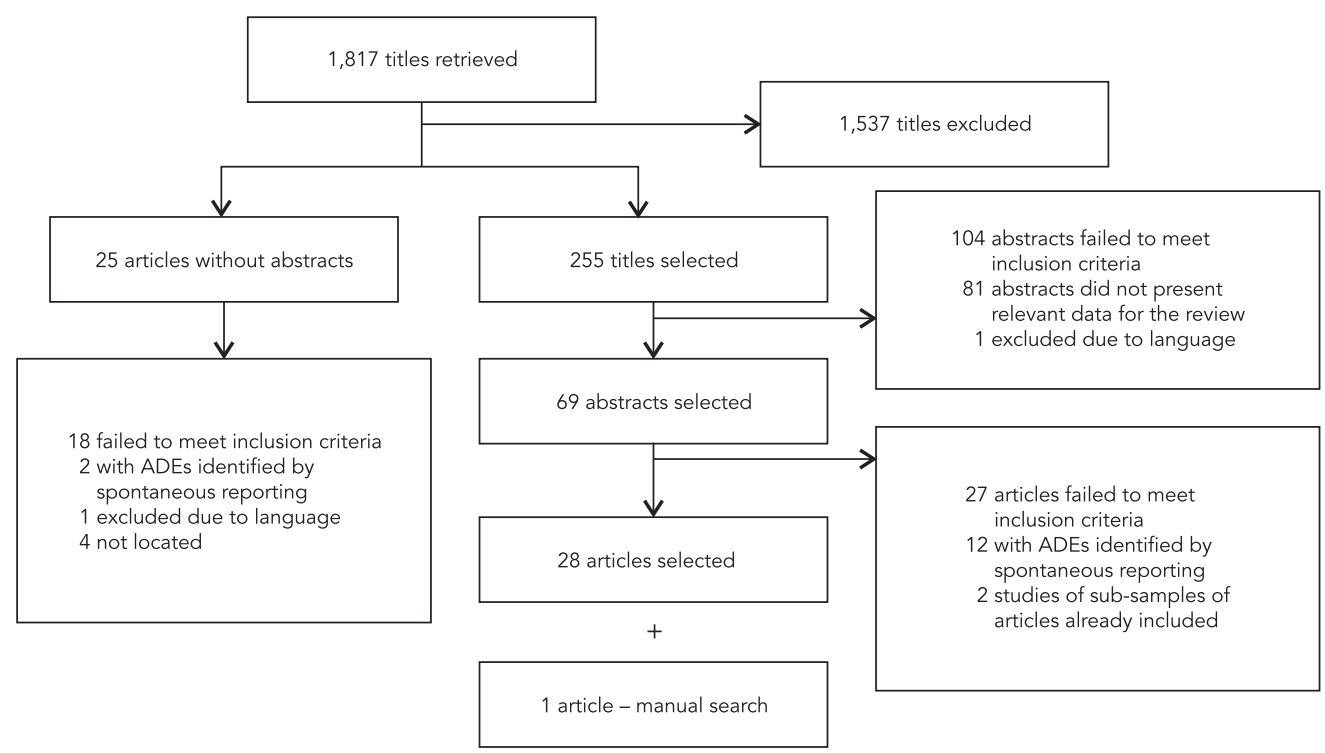

four 10,11,14,30 did not provide information on the place where they were performed. Interestingly, $62.1 \%$ of the studies (18/29) 6,7,8,9,12,13,15,16,19,20,21, $22,25,26,27,31,33,34$ involved university, academic, or teaching hospitals, i.e., presumably large-scale, high-complexity hospital units.

Forty-eight percent of the studies (14/29) 6,8, $9,14,18,19,22,23,24,25,28,29,30,31$ used a retrospective approach, i.e., the presence of ADEs was analyzed after the patient was discharged; $48.3 \%$ of the studies (14/29) 7,11,12,13,15,16,17,20,21,26,27,32,33,34 used a prospective approach, whereby the ADEs were identified during the hospital stay; and one study 10 was cross-sectional, where ADEs were identified using a survey. Among the studies with a prospective approach, two used retrospective strategies to complement the evaluation, based on an analysis of hospital discharge forms 16,21.

All the studies described the target population's size. Only $41.4 \%(12 / 29) 6,7,10,11,12,20,21,26,27$, $28,29,33$ provided information about age, and $24.1 \%$ ( $7 / 29) 6,7,10,20,26,27,33$ on gender. As for the unit of analysis, $62.1 \%(18 / 29) 6,7,10,11,12,15,18,20,21,24,25,26$, $27,30,31,32,33,34$ used the number of patients or patient charts, $48.3 \%$ (14/29) 6,8,9,12,14,16,17,20,22,23,25, $28,29,33$ the number of admissions or discharges, and $24.1 \%(7 / 29) 13,16,17,19,31,32,34$ the number of patient-days.
As for the technique used to identify ADEs, $55.2 \%$ of the studies (16/29) $6,7,8,9,11,13,15,16,17,19$, $21,24,25,26,27,33$ used a combination of strategies to capture the events. The most widely used techniques included monitoring with screening criteria, review of patient charts, and use of the International Classification of Diseases (ICD) associated with ADEs. Monitoring with screening criteria related to the events was performed by $34.5 \%$ of the studies (10/29) 7,9,13,15,18,19,22,26,28,31, either alone (3/29) $18,22,28$ or in association (7/29) $7,9,13,15,19,26,31$. The filters consisted basically of rescue drugs, altered laboratory findings, or signs and symptoms related to adverse events. Thirtyeight per cent (11/29) 8,9,13,14,15,17,19,24,30,31,33 of the studies used patient chart review, while in four of these 12,14,30,31 the patient charts were only reviewed to confirm the events. Twenty-eight per cent $(8 / 29) 6,14,21,23,24,25,29,30$ of the studies identified the ICD codes related to the events, of which four studies used ICD-9 14,21,23,30, three ICD-10 6,25,29, and one the clinically modified ICD-9 24 .

Other techniques included spontaneous reporting, stimulated reporting, review of lists of interventions recorded by staff pharmacists, staff interviews, and medical and pharmaceutical visits. 
Table 1

Characteristics of studies on adverse drug events (ADEs) among patients in general hospitals.

\begin{tabular}{|c|c|c|c|c|c|c|}
\hline Reference & Country/Year & Hospital's characteristic & Design & $\begin{array}{l}\text { Population's } \\
\text { characteristics }\end{array}$ & $\begin{array}{c}\text { Techniques for } \\
\text { identification of } \\
\text { ADEs }\end{array}$ & Frequency \\
\hline $\begin{array}{l}\text { Lagnaoui } \\
\text { et al. } 34\end{array}$ & France/2000 & University hospital & Prospective & $\begin{array}{l}\mathrm{N}=444 \text { patients; } \\
2,569 \text { patient-days }\end{array}$ & $\begin{array}{l}\text { Medical and } \\
\text { pharmaceutical visit }\end{array}$ & $\begin{array}{l}4.7 \% \text { of patients } \\
\text { with ADEs; } 10.1 \\
\text { ADEs per } 1,000 \\
\text { patient-days; } 5.9 \\
\text { ADEs per } 100 \\
\text { hospitalizations }\end{array}$ \\
\hline $\begin{array}{l}\text { Fattinger } \\
\text { et al. } 6\end{array}$ & Switzerland/2000 & $\begin{array}{l}2 \text { teaching hospitals (one } \\
\text { tertiary, one secondary) }\end{array}$ & Retrospective & $\begin{array}{c}\mathrm{N}=3,624 \text { patients; } \\
4,331 \text { hospitalizations } \\
\text { Age: median female } \\
64 \text { (46-71), median } \\
\text { male } 59 \text { (46-77) } \\
\text { Males } 40.6 \%\end{array}$ & $\begin{array}{l}\text { Monitoring using } \\
\text { computerized } \\
\text { database - drugs, } \\
\text { clinical events, lab } \\
\text { results, ICD-10, and } \\
\text { physician evaluation }\end{array}$ & $\begin{array}{c}48 \% \text { of } \\
\text { hospitalizations } \\
\text { with events } \\
\text { possibly related } \\
\text { to drug; } 41 \% \text { of } \\
\text { hospitalizations } \\
\text { with events } \\
\text { possibly related to } \\
\text { drugs, not related } \\
\text { to the disease }\end{array}$ \\
\hline $\begin{array}{l}\text { Dormann } \\
\text { et al. } 7\end{array}$ & Germany/2000 & $\begin{array}{l}\text { University hospital ( } 9 \\
\text { beds) }\end{array}$ & Prospective & $\begin{array}{c}\mathrm{N}=379 \text { patients } \\
\text { Mean age } 50.8(17-88) \\
\text { Males } 6.7 \%\end{array}$ & $\begin{array}{l}\text { Stimulated } \\
\text { spontaneous } \\
\text { reporting and } \\
\text { screening criteria } \\
\text { (lab data) }\end{array}$ & $\begin{array}{l}11.8 \% \text { of patients } \\
\text { with ADEs }\end{array}$ \\
\hline Suh et al. 8 & USA/2000 & University hospital & Retrospective & $\begin{array}{c}\mathrm{N}=9,311 \\
\text { hospitalizations }\end{array}$ & $\begin{array}{l}\text { Spontaneous } \\
\text { reporting and review } \\
\text { of patient charts }\end{array}$ & $\begin{array}{c}1.6 \% \text { of } \\
\text { hospitalizations } \\
\text { with ADEs * }\end{array}$ \\
\hline Senst et al. 9 & USA/2001 & $\begin{array}{l}1 \text { University hospital; } 3 \\
\text { hospitals (mental health } \\
\text { and pediatrics) }\end{array}$ & Retrospective & $\begin{array}{c}\mathrm{N}=3,187 \\
\text { hospitalizations }\end{array}$ & $\begin{array}{l}\text { Screening criterion } \\
\text { (medication, lab data, } \\
\text { and combination } \\
\text { of data), review of } \\
\text { pharmaceutical and } \\
\text { medical records and } \\
\text { patient chart sample }\end{array}$ & $\begin{array}{l}\text { 4.2 ADEs per } 100 \\
\text { hospitalizations ** }\end{array}$ \\
\hline $\begin{array}{l}\text { Baune } \\
\text { et al. } 10\end{array}$ & France/2003 & NS & $\begin{array}{l}\text { Cross- } \\
\text { sectional }\end{array}$ & $\begin{array}{c}\mathrm{N}=902 \text { patients } \\
\text { Mean age 61years } \\
\text { Males } 48.8 \%\end{array}$ & Staff survey & $\begin{array}{c}6.3 \%(4.7 \%-7.9 \%) \\
\text { of patients with } \\
\text { ADEs }\end{array}$ \\
\hline $\begin{array}{l}\text { Ramesh } \\
\text { et al. } 11\end{array}$ & India/2003 & NS & Prospective & $\begin{array}{c}\mathrm{N}=3,717 \text { patients } \\
\text { Age: children } 22.8 \%, \\
\text { adults } 44.3 \% \text {, elderly } \\
32.9 \%\end{array}$ & $\begin{array}{l}\text { Spontaneous } \\
\text { reporting and } \\
\text { stimulated by } \\
\text { pharmacist }\end{array}$ & $\begin{array}{l}3.7 \% \text { of patients } \\
\text { with ADEs ** }\end{array}$ \\
\hline $\begin{array}{l}\text { Dormann } \\
\text { et al. } 12\end{array}$ & Germany/2004 & University hospital & Prospective & $\begin{array}{c}\mathrm{N}=630 \text { patients; } 844 \\
\text { hospitalizations } \\
\text { Median age } 57(18-97)\end{array}$ & $\begin{array}{l}\text { Patient monitoring } \\
\text { with patient chart } \\
\text { review (signs and } \\
\text { symptoms, lab data) }\end{array}$ & $\begin{array}{c}15.2 \% \text { of } \\
\text { hospitalizations } \\
\text { with ADEs; } 23.3 \\
\text { ADEs per } 100 \\
\text { hospitalizations }\end{array}$ \\
\hline $\begin{array}{l}\text { Forster et } \\
\text { al. } 13\end{array}$ & Canada/2004 & $\begin{array}{c}\text { Academic hospital (30 } \\
\text { beds) }\end{array}$ & Prospective & $\mathrm{N}=543$ patient-days & $\begin{array}{l}\text { Patient monitoring: } \\
\text { staff interview, } \\
\text { patient chart } \\
\text { review, screening } \\
\text { criteria, and review } \\
\text { of spontaneous } \\
\text { reporting of errors }\end{array}$ & $\begin{array}{l}\text { 4.4 ADEs per } 100 \\
\text { patient-days }\end{array}$ \\
\hline $\begin{array}{l}\text { Corral } \\
\text { Baena } 14\end{array}$ & Spain/2004 & NS & Retrospective & $\mathrm{N}=32,253$ discharges & $\begin{array}{l}\text { Secondary database } \\
\text { of discharge forms } \\
\text { - use of ICD-9 with } \\
\text { patient chart review }\end{array}$ & $\begin{array}{l}2.15 \% \text { of discharge } \\
\text { forms with } \\
\text { ADEs } \star \star \star\end{array}$ \\
\hline $\begin{array}{l}\text { Weingart } \\
\text { et al. } 15\end{array}$ & USA/2004 & $\begin{array}{c}\text { Teaching hospital (40 } \\
\text { beds) }\end{array}$ & Prospective & $\mathrm{N}=209$ patients & $\begin{array}{c}\text { Spontaneous } \\
\text { reporting, review } \\
\text { of intervention } \\
\text { of pharmacists } \\
\text { staff, reports with } \\
\text { confidential staff } \\
\text { interviews, screening } \\
\text { criteria }\end{array}$ & $\begin{array}{l}5.3 \% \text { of patients } \\
\text { with ADEs; } 7.7 \% \\
\text { of patients with } \\
\text { "close calls" }\end{array}$ \\
\hline
\end{tabular}

(continues) 
Table 1 (continued)

\begin{tabular}{|c|c|c|c|c|c|c|}
\hline Reference & Country/Year & Hospital's characteristic & Design & $\begin{array}{l}\text { Population's } \\
\text { characteristics }\end{array}$ & $\begin{array}{l}\text { Techniques for } \\
\text { identification of } \\
\text { ADEs }\end{array}$ & Frequency \\
\hline $\begin{array}{l}\text { Nebeker } \\
\text { et al. } 16\end{array}$ & USA/2005 & Teaching hospital, tertiary & Prospective & $\begin{array}{l}\mathrm{N}=937 \\
\begin{array}{c}\text { hospitalizations; } 6,856 \\
\text { patient-days }\end{array}\end{array}$ & $\begin{array}{l}\text { Patient monitoring: } \\
\text { review of notes, } \\
\text { prescription, results } \\
\text { of lab tests, and } \\
\text { discharge summary }\end{array}$ & $\begin{array}{l}70 \text { ADEs per } 1,000 \\
\text { patient-days; } 52 \\
\text { ADEs per } 100 \\
\text { hospitalizations; } \\
25 \% \text { of patients } \\
\text { with ADEs }\end{array}$ \\
\hline $\begin{array}{l}\text { Al-Tajir \& } \\
\text { Kelly } 17\end{array}$ & $\begin{array}{l}\text { United Arab } \\
\text { Emirates/2005 }\end{array}$ & Tertiary hospital & Prospective & $\begin{array}{c}N=5,235 \\
\text { hospitalizations; } \\
37,360 \text { patient-days }\end{array}$ & $\begin{array}{l}\text { Spontaneous } \\
\text { reporting } \\
\text { Monitoring: lab } \\
\text { data, nursing notes, } \\
\text { patient chart review, } \\
\text { and spontaneous } \\
\text { reporting }\end{array}$ & $\begin{array}{l}0.07 \text { ADEs per } \\
100 \text { patient-days } \\
\text { (spontaneous } \\
\text { reporting) }{ }^{\star \star *} ; \\
3.59 \text { ADEs per } \\
100 \text { patient-days } \\
\text { (monitoring) }{ }^{* \star *}\end{array}$ \\
\hline $\begin{array}{l}\text { Cohen } \\
\text { et al. } 18\end{array}$ & USA/2005 & Community hospital & Retrospective & $\begin{array}{c}\mathrm{N}=120 \text { patient charts } \\
\text { (baseline), } 90 \text { patient } \\
\text { charts (transition), and } \\
370 \text { patient charts } \\
\text { (post-intervention) }\end{array}$ & Screening criteria & 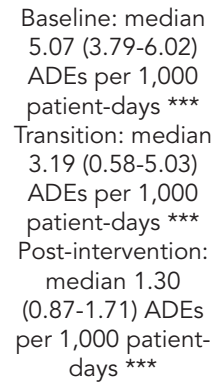 \\
\hline $\begin{array}{l}\text { Mycyk } \\
\text { et al. } 19\end{array}$ & USA/2005 & $\begin{array}{c}\text { Academic hospital, } \\
\text { tertiary }\end{array}$ & Retrospective & $\begin{array}{c}\mathrm{N}=150,973 \\
\text { patient-days \# (pre- } \\
\text { intervention), } 160,748 \\
\text { patient-days \# (post- } \\
\text { intervention) }\end{array}$ & $\begin{array}{l}\text { Electronic database: } \\
\text { screening criteria } \\
\text { (medication and lab } \\
\text { results), spontaneous } \\
\text { reporting and patient } \\
\text { chart review }\end{array}$ & $\begin{array}{c}\text { Pre-intervention: } \\
\text { 1.3 ADEs per } \\
\text { 1,000 patient- } \\
\text { days } * \star \star \\
\text { Post-intervention: } \\
\text { 1.1 ADEs per } \\
\text { 1,000 patient- } \\
\text { days } * \star \star\end{array}$ \\
\hline $\begin{array}{l}\text { Camargo } \\
\text { et al. } 20\end{array}$ & Brazil/2006 & University hospital & Prospective & $\begin{array}{c}\mathrm{N}=333 \text { patients; } 335 \\
\text { hospitalizations } \\
\text { Mean age } 52.3(\mathrm{SD}= \\
17.85) \\
\text { Males } 45.1 \%\end{array}$ & $\begin{array}{l}\text { Intensive monitoring: } \\
\text { use of patient } \\
\text { chart as source of } \\
\text { information }\end{array}$ & $\begin{array}{l}25.9 \%(21.0 \%- \\
30.7 \%) \text { of patients } \\
\text { with ADEs \#\# }\end{array}$ \\
\hline $\begin{array}{l}\text { Otero-Lopez } \\
\text { et al. } 21\end{array}$ & Spain/2006 & University hospital & $\begin{array}{c}\text { Prospective } \\
\text { \#\#\# }\end{array}$ & $\begin{array}{c}\mathrm{N}=2,643 \text { patients } \\
\text { Mean age } 71.7(18-93)\end{array}$ & $\begin{array}{l}\text { Discharge forms: use } \\
\text { of ICD-9 in secondary } \\
\text { diagnosis and review } \\
\text { of clinical history. } \\
\text { Stimulated reporting }\end{array}$ & $\begin{array}{c}7.2 \%(6.2 \%-8.2 \%) \\
\text { of patients with } \\
\text { ADEs }\end{array}$ \\
\hline $\begin{array}{l}\text { Kilbridge } \\
\text { et al. } 22\end{array}$ & USA/2006 & $\begin{array}{l}1 \text { community and } 1 \\
\text { university hospital }\end{array}$ & Retrospective & $\begin{array}{c}\mathrm{N}=25,177 \\
\text { hospitalizations } \\
\text { (university hospital), } \\
8,029 \text { hospitalizations } \\
\text { (community hospital) }\end{array}$ & $\begin{array}{l}\text { Electronic database: } \\
\text { screening criteria }\end{array}$ & $\begin{array}{l}\text { University hospital: } \\
\text { 4.4 ADEs per } 100 \\
\text { hospitalizations } \\
\text { Community } \\
\text { hospital: } 6.2 \\
\text { ADEs per } 100 \\
\text { hospitalizations *** }\end{array}$ \\
\hline $\begin{array}{l}\text { Bond \& } \\
\text { Raehl } 23\end{array}$ & USA/2006 & 3,328 hospitals & Retrospective & $\begin{array}{c}\mathrm{N}=8,067,562 \\
\text { hospitalizations }\end{array}$ & $\begin{array}{c}\text { Secondary database } \\
\text { of discharge forms: } \\
\text { use of ICD-9 }\end{array}$ & $\begin{array}{c}1.7 \% \text { of } \\
\text { hospitalizations } \\
\text { with ADEs *** }\end{array}$ \\
\hline $\begin{array}{l}\text { Hougland } \\
\text { et al. } 24\end{array}$ & USA/2006 & 41 acute care hospitals & Retrospective & $\begin{array}{c}\mathrm{N}=1,961 \text { patient } \\
\text { charts }\end{array}$ & $\begin{array}{l}\text { Discharge forms: } \\
\text { use of ICD-9-CM in } \\
\text { secondary diagnosis } \\
\text { and E-code with } \\
\text { patient chart review }\end{array}$ & $\begin{array}{l}\text { 3.6 ADEs per } 100 \\
\text { patient charts } \S\end{array}$ \\
\hline $\begin{array}{l}\text { Lugardon } \\
\text { et al. } 25\end{array}$ & France/2006 & Teaching hospital & Retrospective & $\begin{array}{l}\mathrm{N}=27,426 \\
\text { patients; } 39,441 \\
\text { hospitalizations }\end{array}$ & $\begin{array}{l}\text { Spontaneous } \\
\text { reporting and } \\
\text { secondary database } \\
\text { of discharge forms: } \\
\text { use of ICD-10; } \\
\text { capture/ recapture }\end{array}$ & 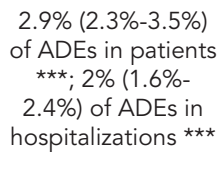 \\
\hline
\end{tabular}

(continues) 
Table 1 (continued)

\begin{tabular}{|c|c|c|c|c|c|c|}
\hline Reference & Country/Year & Hospital's characteristic & Design & $\begin{array}{l}\text { Population's } \\
\text { characteristics }\end{array}$ & $\begin{array}{c}\text { Techniques for } \\
\text { identification of } \\
\text { ADEs }\end{array}$ & Frequency \\
\hline $\begin{array}{l}\text { Davies } \\
\text { et al. } 26\end{array}$ & England/2006 & University hospital & Prospective & $\begin{array}{c}\mathrm{N}=125 \text { patients } \\
\text { Median age (patients } \\
\text { with ADEs) } 69.5(52- \\
\text { 79); median (patients } \\
\text { without ADEs) } 61 \\
\text { (45-78) } \\
\text { Males 51.2\% }\end{array}$ & $\begin{array}{l}\text { Pharmaceutical } \\
\text { visit: screening } \\
\text { criteria (medication), } \\
\text { spontaneous } \\
\text { reporting and } \\
\text { evaluation of new } \\
\text { symptoms }\end{array}$ & $\begin{array}{c}19.2 \%(12 \%-26 \%) \\
\text { of patients with } \\
\text { ADEs }\end{array}$ \\
\hline $\begin{array}{l}\text { Tribino } \\
\text { et al. } 27\end{array}$ & Colombia/2006 & $\begin{array}{l}\text { University hospital, } \\
\text { tertiary ( } 42 \text { beds) }\end{array}$ & Prospective & $\begin{array}{c}\mathrm{N}=836 \text { patients } \\
\text { Mean age } 58.9(\mathrm{SD} \\
0.67) \\
\text { Males } 54 \%\end{array}$ & $\begin{array}{l}\text { Spontaneous } \\
\text { reporting and review } \\
\text { of clinical history } \\
\text { (signs and symptoms, } \\
\text { physical examination, } \\
\text { and patient interview, } \\
\text { confirmed by } \\
\text { clinician) }\end{array}$ & $\begin{array}{l}25.1 \% \text { of patients } \\
\text { with ADEs; } 32 \\
\text { ADEs per } 100 \\
\text { patients }\end{array}$ \\
\hline $\begin{array}{l}\text { Schade } \\
\text { et al. } 28\end{array}$ & USA/2006 & Rural acute care hospital & Retrospective & $\begin{array}{c}N=3,572 \text { discharges } \\
\text { Age }>18 \text { years }\end{array}$ & $\begin{array}{l}\text { Screening criterion } \\
\text { (rescue drugs) }\end{array}$ & $\begin{array}{c}1.73 \% \text { of } \\
\text { discharges with } \\
\text { ADEs } \S \S\end{array}$ \\
\hline Rozenfeld 29 & Brazil/2007 & $\begin{array}{l}\text { Hospitals accredited by SUS } \\
\text { in Rio de Janeiro }\end{array}$ & Retrospective & $\begin{array}{c}\mathrm{N}=1,898,676 \\
\text { discharges } \\
\text { Age } \geq 20 \text { years }\end{array}$ & $\begin{array}{l}\text { Secondary database of } \\
\text { discharge forms: use of } \\
\text { ICD-10 }\end{array}$ & $\begin{array}{l}1.8 \text { cases per } 1,000 \\
\text { hospitalizations } * \star \star\end{array}$ \\
\hline $\begin{array}{l}\text { Sanchez- } \\
\text { Muñoz, } \\
\text { et al. } 30\end{array}$ & Spain/2007 & NS & Retrospective & $\mathrm{N}=3,983$ patients & $\begin{array}{l}\text { Secondary database } \\
\text { of discharge forms: } \\
\text { use of ICD-9 with } \\
\text { patient chart review }\end{array}$ & $\begin{array}{l}2.2 \% \text { of patients } \\
\text { with ADEs }\end{array}$ \\
\hline $\begin{array}{l}\text { Hwang } \\
\text { et al. } 31\end{array}$ & South Korea/2008 & Teaching hospital, tertiary & Retrospective & $\begin{array}{l}N=598 \text { patients; } \\
6,578 \text { patient-days }\end{array}$ & $\begin{array}{l}\text { Screening criteria } \\
\text { with patient chart } \\
\text { reviews }\end{array}$ & $\begin{array}{l}31.3 \% \text { ADEs in } \\
\text { patients ***; } 28.4 \\
\text { ADEs per } 1,000 \\
\text { patient-days } * \star \star\end{array}$ \\
\hline $\begin{array}{l}\text { Pourseyed } \\
\text { et al. } 32\end{array}$ & Iran/2009 & $\begin{array}{c}\text { General hospital (35 } \\
\text { beds) }\end{array}$ & Prospective & $\begin{array}{c}\mathrm{N}=400 \text { patients; } \\
3,276 \text { patient-days } \\
\text { Mean age } 60.41 \text { (SD } \\
16.97 \text { ) } \\
\text { Males } 50.8 \%\end{array}$ & $\begin{array}{l}\text { Intensive monitoring: } \\
\text { daily patient follow- } \\
\text { up until discharge }\end{array}$ & $\begin{array}{l}10 \% \text { of patients } \\
\text { with ADEs }\end{array}$ \\
\hline $\begin{array}{l}\text { Davis } \\
\text { et al. } 33\end{array}$ & England/2009 & University hospital & Prospective & $\begin{array}{c}N=3,322 \text { patients; } \\
3,695 \text { hospitalizations }\end{array}$ & $\begin{array}{l}\text { Pharmaceutical visit: } \\
\text { patient chart review, } \\
\text { medical and nursing } \\
\text { notes, lab data, and } \\
\text { direct information } \\
\text { from staff and patient }\end{array}$ & $\begin{array}{c}15.8 \% \text { of patients } \\
\text { with ADEs; } 14.7 \% \\
\text { (13.3\%-15.9\%) of } \\
\text { hospitalizations } \\
\text { with ADEs }\end{array}$ \\
\hline
\end{tabular}

ICD-9: International Classification of Diseases - 9th revision; ICD-9-CM: International Classification of Diseases - 9th revision, clinically modified; ICD-10: International Classification of Diseases - 10th revision; SD: standard deviation; NS: not specified; SUS: Unified National Health System (Brazil).

* Rate of patients with events was calculated by dividing the number of patients with ADEs by the number of hospitalizations;

** Rate includes pediatric patients;

*** Does not distinguish between events that occurred before or during hospitalization;

\# Number of patient-days was calculated by multiplying the ADE rate by the number of events;

\#\# Event occurred before or during the hospitalization;

\#\#\# Prospective approaches retrieved 182 events and retrospective approaches 9 events. Eight events were retrieved by both approaches;

$\S$ Rate was calculated by dividing the number of events by the number of patient charts reviewed;

$\S \S$ Frequency of discharges with ADEs was calculated by dividing the number of discharges with at least one ADE by the number of discharges.

As for the estimates of frequency of events, the indicators varied. In addition, ten studies $14,17,18,19,20,22,23,25,29,31$ did not present estimates that differentiated between events leading to hospital admission and those that occurred before or during hospitalization. One study ${ }^{29}$ called attention to the fact that such differentiation was impossible, due to the method used to identify events. Table 2 lists the studies that focused on events that occurred during hospitalization, with the respective frequency estimates. The events estimator is the proportion of patients or hospitalizations with ADEs. Readmissions were considered independent events, based on Dormann et al. 12, whose findings showed that the occurrence of an adverse drug event in one hospitalization is not a predictive factor for readmission. Another estimator is the rate of events per 100 
Table 2

Characteristics of studies that evaluated adverse drug events (ADEs) that occurred exclusively during stay in general hospitals

\begin{tabular}{|c|c|c|c|c|c|c|}
\hline Reference/Year & $\begin{array}{c}\text { Techniques for } \\
\text { identification of ADEs }\end{array}$ & $\begin{array}{c}\text { Proportion of } \\
\text { patients or } \\
\text { hospitalizations } \\
\text { (\%) }\end{array}$ & $\begin{array}{c}\text { ADEs/100 } \\
\text { patients or } \\
\text { hospitalizations }\end{array}$ & $\begin{array}{l}\text { ADEs/100 } \\
\text { patient-days }\end{array}$ & Severity & Avoidability \\
\hline $\begin{array}{l}\text { Lagnaoui } \\
\text { et al. } 34 / 2000\end{array}$ & $\begin{array}{c}\text { Physician and pharmacist } \\
\text { visit }\end{array}$ & 4.7 & 10.1 & 5.9 & Serious $7.7 \%$ & $\begin{array}{l}\text { Avoidable } \\
50 \%\end{array}$ \\
\hline $\begin{array}{l}\text { Fattinger et al. } \\
6 / 2000\end{array}$ & $\begin{array}{l}\text { Monitoring using } \\
\text { electronic database: } \\
\text { drugs, clinical events, } \\
\text { lab results, ICD-10, and } \\
\text { evaluation by clinician }\end{array}$ & 41.4 & NS & NS & NS & NS \\
\hline $\begin{array}{l}\text { Dormann } \\
\text { et al. } 7 / 2000\end{array}$ & $\begin{array}{l}\text { Stimulated spontaneous } \\
\text { reporting and screening } \\
\text { criteria (lab data) }\end{array}$ & 11.8 & 12.1 & NS & $\begin{array}{c}\text { Mild } 48 \% \text {; moderate } 46 \% ; \\
\text { serious } 6 \%\end{array}$ & NS \\
\hline Suh et al. $8 / 2000$ & $\begin{array}{l}\text { Spontaneous reporting } \\
\text { and patient chart review }\end{array}$ & 1.6 & NS & NS & $\begin{array}{c}\text { Mild } 30 \% \text {; moderate } 53 \% ; \\
\text { serious } 17 \%\end{array}$ & NS \\
\hline Senst et al. 9/2001 & $\begin{array}{l}\text { Screening criterion } \\
\text { (medication, lab data, } \\
\text { and combination of data), } \\
\text { review of pharmaceutical } \\
\text { and medical records, } \\
\text { and review of sample of } \\
\text { patient charts }\end{array}$ & NS & 4.2 & NS & $\begin{array}{l}\text { Significant } 36 \% \text {; serious } \\
45 \% \text {; life-threatening } 36 \%\end{array}$ & $\begin{array}{c}\text { Avoidable } \\
14.8 \%\end{array}$ \\
\hline Baune et al. 10/2003 & Staff interview & 6.3 & NS & NS & Serious or severe $73 \%$ & $\begin{array}{l}\text { Avoidable } \\
25 \%\end{array}$ \\
\hline Ramesh et al. 11/2003 & $\begin{array}{c}\text { Spontaneous reporting, } \\
\text { and stimulated by } \\
\text { pharmacist }\end{array}$ & 3.7 & NS & NS & NS & NS \\
\hline $\begin{array}{l}\text { Dormann } \\
\text { et al. } 12 / 2004\end{array}$ & $\begin{array}{l}\text { Patient monitoring and } \\
\text { patient chart review (signs } \\
\text { and symptoms, lab data) }\end{array}$ & 15.2 & 23.3 & NS & NS & NS \\
\hline Forster et al. 13/2004 & $\begin{array}{c}\text { Patient monitoring: } \\
\text { staff interview, patient } \\
\text { chart review, screening } \\
\text { criteria, and review of } \\
\text { spontaneous reporting } \\
\text { of errors }\end{array}$ & NS & NS & 4.4 & $\begin{array}{l}\text { Significant 42\%; serious } \\
46 \% \text {; life-threatening 13\% }\end{array}$ & $\begin{array}{c}\text { Avoidable } \\
2.6 \mathrm{ADEs} / 100 \\
\text { patient-days }\end{array}$ \\
\hline $\begin{array}{l}\text { Weingart } \\
\text { et al. } 15 / 2004\end{array}$ & $\begin{array}{c}\text { Spontaneous reporting, } \\
\text { review of intervention of } \\
\text { pharmacists staff, reports } \\
\text { with confidential staff } \\
\text { interviews, screening } \\
\text { criteria }\end{array}$ & 5.3 & NS & NS & $\begin{array}{c}\text { Significant } 54.5 \% \text {; serious } \\
27.3 \% \text {; life-threatening } \\
18.2 \%\end{array}$ & $\begin{array}{l}\text { Probably } \\
\text { avoidable } \\
27.3 \%\end{array}$ \\
\hline $\begin{array}{l}\text { Nebeker } \\
\text { et al. 16/2005 }\end{array}$ & $\begin{array}{l}\text { Patient monitoring: review } \\
\text { of notes, prescription, } \\
\text { results of lab tests, and } \\
\text { discharge summary }\end{array}$ & 25.7 & 51.5 & 7.0 & $\begin{array}{c}\text { Moderate 91\%; serious } \\
9 \% \text {; level E } 87 \% \text { *; level F } \\
4 \% \text { *; level G < 1\%*; level } \\
\text { H 3\% *; level I 6\%* }\end{array}$ & NS \\
\hline $\begin{array}{l}\text { Otero-Lopez } \\
\text { et al. } 21 / 2006\end{array}$ & $\begin{array}{c}\text { Discharge forms: use } \\
\text { of ICD-9 in secondary } \\
\text { diagnosis and review of } \\
\text { clinical history. Stimulated } \\
\text { reporting }\end{array}$ & 7.2 & NS & NS & $\begin{array}{l}\text { Mild } 63.3 \% \text {; moderate } \\
30.4 \% \text {; serious } 6.3 \%\end{array}$ & $\begin{array}{c}\text { Avoidable } \\
19.9 \%\end{array}$ \\
\hline $\begin{array}{l}\text { Hougland } \\
\text { et al. } 24 / 2006\end{array}$ & $\begin{array}{l}\text { Discharge forms: use of } \\
\text { ICD-9-CM in secondary } \\
\text { diagnosis and E-code with } \\
\text { patient chart reviews }\end{array}$ & NS & 3.6 & NS & NS & NS \\
\hline Davies et al. 26/2006 & $\begin{array}{l}\text { Screening criteria } \\
\text { (medication), spontaneous } \\
\text { reporting and evaluation } \\
\text { of new symptoms }\end{array}$ & 19.2 & NS & NS & $\begin{array}{l}\text { Level } 366.6 \% \text { **; level } 4 \\
26 \% \text { **; level 7a } 7.4 \% \text { ** }\end{array}$ & $\begin{array}{c}\text { Possible } \\
\text { avoidable } \\
48 \% ; \\
\text { definitely } \\
\text { avoidable } \\
11 \%\end{array}$ \\
\hline
\end{tabular}

(continues) 
Table 2 (continued)

\begin{tabular}{|c|c|c|c|c|c|c|}
\hline Reference/Year & $\begin{array}{c}\text { Techniques for } \\
\text { identification of ADEs }\end{array}$ & $\begin{array}{c}\text { Proportion of } \\
\text { patients or } \\
\text { hospitalizations } \\
\text { (\%) }\end{array}$ & $\begin{array}{c}\text { ADEs/100 } \\
\text { patients or } \\
\text { hospitalizations }\end{array}$ & $\begin{array}{l}\mathrm{ADEs} / 100 \\
\text { patient-days }\end{array}$ & Severity & Avoidability \\
\hline Tribino et al. 27/2006 & $\begin{array}{l}\text { Spontaneous reporting } \\
\text { and review of clinical } \\
\text { history (signs and } \\
\text { symptoms, physical } \\
\text { examination, and patient } \\
\text { interview, confirmed by } \\
\text { clinician) }\end{array}$ & 25.1 & 32.1 & NS & $\begin{array}{c}\text { Mild } 13.4 \% \text {; moderate } \\
81.3 \% \text {; serious } 4.1 \% \text {; fatal } \\
1.1 \%\end{array}$ & NS \\
\hline Schade et al. 28/2006 & $\begin{array}{c}\text { Screening criterion (rescue } \\
\text { drugs) }\end{array}$ & NS & 1.7 & NS & NS & $\begin{array}{l}\text { Avoidable } \\
25.8 \%\end{array}$ \\
\hline $\begin{array}{l}\text { Sanchez-Muñoz } \\
\text { et al. 30/2007 }\end{array}$ & $\begin{array}{c}\text { Secondary database of } \\
\text { discharge forms: use of } \\
\text { ICD-9 with patient chart } \\
\text { review }\end{array}$ & 2.2 & NS & NS & $\begin{array}{l}\text { Mild } 38.6 \% \text {; moderate } \\
58 \% \text {; serious } 3.4 \%\end{array}$ & $\begin{array}{l}\text { Avoidable } \\
52.1 \%\end{array}$ \\
\hline $\begin{array}{l}\text { Pourseyed } \\
\text { et al. 32/2008 }\end{array}$ & $\begin{array}{l}\text { Intensive monitoring: daily } \\
\text { patient follow-up until } \\
\text { discharge }\end{array}$ & $10 \%$ & NS & NS & NS & NS \\
\hline Davies et al. 33/2009 & $\begin{array}{l}\text { Pharmaceutical visit: } \\
\text { patient chart review, } \\
\text { medical and nursing } \\
\text { notes, lab data, and direct } \\
\text { information from staff and } \\
\text { patient }\end{array}$ & 15.8 & NS & NS & 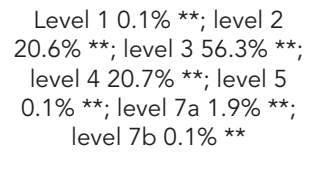 & $\begin{array}{c}\text { Possibly } \\
\text { avoidable } \\
46.9 \% ; \\
\text { definitely } \\
\text { avoidable } \\
6.4 \%\end{array}$ \\
\hline
\end{tabular}

ICD-9: International Classification of Diseases - 9th revision; ICD-9-CM: International Classification of Diseases - 9th revision, clinically modified; ICD-10: International Classification of Diseases - 10th revision; NS: not specified.

* The severity of the event increases from level $E$ to $I$, where level I is the patient's death;

** The severity of the event increases from level 1 to $7 \mathrm{~b}$, where events classified as $7 \mathrm{~b}$ are those directly related to the patient's death.

patients or admissions, or the rate of events per 100 patient-days.

The proportion of patients or admissions with ADEs during hospitalization (Table 2) varied from 1.6 to $41.4 \%$, and the rates ranged from 1.7 to 51.5 events per 100 hospitalizations and from 4.4 to 7.0 events per 100 patient-days.

Of the 19 studies, $68.4 \%$ (13/19) 7,8,9,10,13,15, $16,21,26,27,30,33,34$ classified the events according to severity. There was a predominance of less serious events, mostly classified as mild or moderate (more than $80 \%$ of total), regardless of the classification used for severity. Fifty-three per cent of the studies (10/19) 9,10,13,15,21,26,28,30,33,34 assessed avoidability. Events were considered avoidable when the authors classified them as avoidable or possibly, definitely, or probably avoidable. The frequency of these events varied from $14.8 \%$ to $59 \%$.

As for the drug classes involved, only eight studies (8/19) 6,7,8,9,16,21,27,30 conducted this analysis. Of the five classes most commonly involved, anti-infective agents were the most frequent, with 8 to $39 \%$ of the reported events. Another class that called attention was cardiovascular agents, related to $25.3 \%$ of the events. Antineoplastics agents were among the five drug classes most frequently related to ADEs and can be attributable in up to $30.7 \%$ of events.

\section{Discussion}

The estimates found in the studies show that medicines used during hospitalization can frequently lead to ADEs, and that a considerable proportion of such events are avoidable.

\section{Variability of studies and the impact on estimates}

This systematic literature review included studies that evaluated ADEs occurring during hospitalization, excluding studies that only used spontaneous reporting as the method for identifying ADEs, in addition to a series of other factors in order to allow analysis of homogeneous studies. Even so, there was a wide range in the frequency of events, which could be explained by the numerous factors related to the studies' characteristics, despite the similar profile of hospitals, patients, medicines prescribed, and disease severity. 
The variability hindered obtaining a summary measure for frequency of ADEs. In addition, nine studies did not differentiate between events that occurred during hospitalization and those occurring prior to it. The disease conditions, drug use pattern, and type of care in and out of hospital differed considerable, which made it impossible to consider events that occurred before or during hospitalization in the same group. Even among the studies that only evaluate events that occur during hospitalization, three distinct event estimators were identified, thus hindering comparison of the studies.

\section{Concepts and definitions}

The definitions used to characterize drug-related harm varied, thus impacting measurement and hamper comparisons between studies. Rissato et al. 35 discuss the need for standardized terminology in their review of terms and concepts used to characterize the harm caused to patients by medicines. Obviously, although the definitions vary widely, they have in common their central focus on drug-related harm.

Historically, in 1966 the World Health Organization (WHO) defined the term "adverse drug reaction" any response to a drug which is noxious, unintended, and which occurs at doses normally used in man for the prophylaxis, diagnosis, or therapy of disease. In 1972, WHO added the phrase "or for modification of physiological function" to the end of this definition. The two definitions were used by $37.9 \%(11 / 29)$ of the studies $7,8,10,11,12,17,18,20,25,27,32$.

Seven studies used the term "adverse drug event" 9,14,19,21,23,30,31, while two used "adverse drug reaction" 26,33 , based on the concept of harm caused to the patient due to use of the drug in the therapeutic context, while four 9,14,21,30 defined the event as any injury, large or small, caused by the therapeutic use (including non-use) of a drug. Non-use involves other situations, namely, problems with access to the drug, adherence, or the fact that the drug was not prescribed, although necessary.

Six studies 13,15,16,28,29,34 presented a broader definition of ADEs, including in their context the harm caused to patients by non-therapeutic use of the drug. Two of these studies used the term adverse drug event 22,24 and one used adverse drug reaction 6 , but without defining them.

Neither the terminology nor the concepts were homogeneous across the studies. Sixteen studies used the term adverse drug event, of which two used the WHO definition for adverse drug reaction 17,18 . Nine studies used the term adverse drug reaction, and one 29 used the term injury, including cases of self-inflicted lesions and poisonings.

According to Rissato et al. 35, the lack of standardization in the concepts and terms allows confusing the notion of adverse drug reaction with other types of events that occur under distinct circumstances from those in which use of the drug occurs at the normally recommended doses.

Three studies 9,13,15 defined and evaluated potential ADEs, considering errors that could have led to harm but were avoided by interception or chance. Detection of such cases is important, because the triggering mechanism is frequently similar to that of the incidents that lead to harm 36 and is thus related to weaknesses in the quality of prescribing and dispensing process.

\section{Identification technique}

One factor that appears to directly impact the estimates is the technique used to capture the events. Al-Tajir \& Kelly 17 demonstrated the complementary nature of the techniques used to identify events, as did Otero-Lopez et al. 21 . Sixteen studies $(55.2 \%)$ used a combination of strategies to identify events.

Studies that used the International Classification of Diseases (ICD) to capture events from information contained in secondary databases showed the lowest frequencies of events, $2.2 \% 30$ and 1.8 per 1,000 hospitalizations 29 .

Four studies identified the codes related to the events using information from secondary databases. These databases contain a considerable volume of data that are easy to access electronically, with a wide coverage. This technique thus proved more useful for detecting events that led to the hospital admission as compared to those that occurred during the hospital stay 14,30.

The most widely used techniques to identify adverse drug events was monitoring by screening criteria. This technique is based on the hypothesis that the occurrence of criteria is closely related to the harm 37 . Data are collected with the aid of screening criteria that can be automated, with the screening performed electronically 19,22 The screening criteria are used as filters for a preliminary analysis of the information recorded on the patient, thereby providing a more objective and viable alternative to traditional patient chart reviews.

\section{Causality}

Another factor that can impact estimates of ADEs is the way the association between the drug's use and patient harm is measured. Cause-effect di- 
agnosis for ADEs is a complex issue. Most events are nonspecific and can be confused with clinical manifestations of the disease under treatment 38 . Algorithms and decision tables, when adequately applied, allow greater objectivity in establishing the causal relationship.

Eleven studies did not use scales or algorithms to assess causality 9,14,15,18,23,24,25,28, $29,30,34$. The others used five distinct classifications. Among the most widely used strategies are the Naranjo algorithm, the Karch \& Lasagna criteria, and the WHO criteria. In relation to the diversity of strategies for determining causality, Thürmann 39 reports that distinct algorithms and the way they are applied are the main sources of discrepancies between studies.

This fact can be observed in the current review. Even the ten studies that used the Naranjo algorithm did not use its proposed categories (definite, probable, possible, or doubtful) in the same way. Thus, six studies 7,12,20,26,27,33 only included definite, probable, and possible events. One study 22 only considered definite and probable events. Another 17 considered definite, probable, possible, and doubtful events, thereby overestimating ADEs as compared to the other studies. Two studies 8,19 failed to specify the categories they included.

Although algorithms may allow greater objectivity in assessing the causal relationship between use of medicines and adverse effects, some clinical judgment persists, for example, when the algorithm involves the following question: are there alternative causes (other than the medication) which could cause the effects by themselves? Reliability studies could provide useful information on the measures' robustness, indicating to what extent repeated evaluations of the same patient chart (or clinical case) would produce the same results. However, none of the studies identified here examined this issue.

\section{Characteristics of the study population}

Although population differences could explain the variability in the frequency of ADEs, the selected studies mainly focused on overall estimates rather than associations between the events and the populations' characteristics. Still, some studies demonstrated that certain more susceptible age brackets 40,41,42 and female gender could be risk factors for ADEs 43.

Characterization of the populations in terms of race and socioeconomic status was virtually nonexistent in the studies. Physicians' perceptions of patients can vary systematically according to the patient's race, socioeconomic status, and other demographic characteristics, and this difference in perception can influence the treatment options for patients and thus their quality of care 44 . Extrapolating these findings to studies on adverse drug events raises the hypothesis that race and socioeconomic status could influence the degree of susceptibility to the occurrence of avoidable events, since these characteristics could impact the quality of care provided.

Furthermore, in a systematic review, MacDowell et al. 45 demonstrated that different ethnic groups display distinct risks for developing adverse drug reactions in cardiovascular diseases. For example, black patients present a relative risk of 1.5 (95\%CI: 1.2-1.9) of intracranial hemorrhage triggered by antithrombotics, when compared to non-black patients.

Other possible predictive factors for the variability in estimates, like diagnoses, prescription pattern, and number of drugs used, could not be considered in the analysis, since they were not reported homogeneously and constantly.

\section{Characteristics of events during hospitalization}

The review covered information on severity, avoidability, and the drugs attributable in adverse events that occurred during hospitalization.

The proportion of avoidable events varied from $14.8 \%$ to $59 \%$. Despite the variability, avoidable events accounted for an important share of the total, and better knowledge of them could help develop preventive strategies and improve the quality of patient care.

As for the drugs involved, the most frequently reported classes were anti-infective, cardiovascular, and antineoplastics agents. Importantly, three drug groups alone may account for more than $50 \%$ of the adverse events, thus potentially signaling a field for intervention and prevention of ADEs.

\section{Study limitations}

This study's findings should be interpreted with its limitations in mind. First, the articles included in the review were mainly retrieved through a search in the MEDLINE database, which includes more American than European journals. This could explain the larger number of studies performed in the United States; on the other hand, the United States is obviously one of the pioneers in research on ADEs. Secondly, we did not conduct any article search in the gray literature or address any direct requests to authors for unpublished data. 
As for the review's external validity, we focused on studies in general hospitals and highcomplexity hospitals, $61.5 \%$ of which were university, academic, or teaching institutions. Since the nature of the clientele affects the prescription patterns and thus the frequency of events, highcomplexity hospitals could increase the risk of ADEs.

\section{Final remarks}

Adverse drug events in inpatients are not unpredictable or incomprehensible accidents, but events that should be monitored and followed in order to understand where and why they occur. The literature is extensive, but highly diversified as to concepts, objectives, methods, and techniques. Estimates of frequency vary widely, thereby demanding additional effort at in-depth and critical analysis.

The fact that a considerable share of the events are classified as avoidable and mostly related to a limited set of drug classes emphasizes importance of hospitals knowing the pattern of events that occur during hospitalization in order to orient preventive strategies.

There is an evident need for standardization of concepts and definitions for describing the harm caused by the use of medicines, as well as for the development of study protocols for ADEs. Standardized approaches would allow comparison of indicators from different hospitals and improve our understanding of the magnitude of the problem.

\section{Resumo}

O objetivo foi avaliar estudos sobre a ocorrência de eventos adversos a medicamentos (EAM) em hospitais para conhecer as suas freqüências e características, comparando os métodos de identificação e as definições utilizadas para caracterizá-los. A busca foi realizada no MEDLINE e identificou estudos publicados entre 2000 e 2009. Os critérios de inclusão foram estudos em população não selecionada por patologias ou medicamentos específicos e os EAM ocorridos durante a internação. Foram selecionados 29 estudos e encontradas múltiplas fontes de heterogeneidade entre eles, incluindo diferenças nas populações estudadas, nas técnicas de vigilância, nas definições de EAM e nos indicadores. A freqüência de pacientes com EAM está entre 1,6\% e 41,4\% dos pacientes internados e as taxas entre 1,7 e 51,8 eventos/100 internações. Uma parte considerável desses eventos poderia ter sido evitada. Os resultados mostram que EAM em pacientes internados são um problema de saúde pública. Entretanto, são necessários novos estudos de monitoramento desses eventos adversos para a efetiva promoção do uso seguro dos medicamentos.

Monitoramento de Medicamentos; Quimioterapia; Farmacoepidemiologia

\section{Contributors}

F. G. Cano participated in the project's design and elaboration, article selection, data collection, data analysis and interpretation, critical data review, and drafting and approval of the article's final version. S. Rozenfeld contributed to the project's design and elaboration, article selection, data analysis and interpretation, critical data review, and correction and approval of the article's final version.

\section{Acknowledgments}

We wish to thank the Graduate Studies Program in Public Health at the Sergio Arouca National School of Public Health, Oswaldo Cruz Foundation, for having supported this work. 
1. Laporte JR, Tognoni G. Principios de epidemiología del medicamento. 2a Ed. Barcelona: Ediciones Científicas y Técnicas; 1993.

2. Seidl LG, Thornton GF, Smith JW, Cluff LE. Studies on the epidemiology of adverse drug reactions. Bull Johns Hopkins Hosp 1966; 119:299-315.

3. Lazarou J, Pomeranz BH, Corey PN. Incidence of adverse drug reactions in hospitalized patients: a meta-analysis of prospective studies. JAMA 1998; 279:1200-5.

4. Kvasz M, Allen IE, Gordon MJ, Ro EY, Estok R, Olkin I, et al. Adverse drug reactions in hospitalized patients: a critique of a meta-analysis. MedGenMed 2000; 2:E3.

5. Otero MJ, Dominguez-Gil A. Acontecimientos adversos por medicamentos: una patología emergente. Farm Hosp 2000; 24:258-66.

6. Fattinger K, Roos M, Vergeres P, Holenstein C, Kind B, Masche U, et al. Epidemiology of drug exposure and adverse drug reactions in two Swiss departments of internal medicine. Br J Clin Pharmacol 2000; 49:158-67.

7. Dormann H, Muth-Selbach U, Krebs S, CriegeeRieck M, Tegeder I, Schneider HT, et al. Incidence and costs of adverse drug reactions during hospitalisation: computerised monitoring versus stimulated spontaneous reporting. Drug Saf 2000; 22:161-8.

8. Suh DC, Woodall BS, Shin SK, Hermes-De Santis ER. Clinical and economic impact of adverse drug reactions in hospitalized patients. Ann Pharmacother 2000; 34:1373-9.

9. Senst BL, Achusim LE, Genest RP, Cosentino LA, Ford CC, Little JA, et al. Practical approach to determining costs and frequency of adverse drug events in a health care network. Am J Health Syst Pharm 2001; 58:1126-32.

10. Baune B, KesslerV, Patris S, Descamps V, Casalino E Quenon JL, et al. Medicinal iatrogenics in hospitals. A survey on a given day. Presse Med 2003; 32:683-8.

11. Ramesh M, Pandit J, Parthasarathi G. Adverse drug reactions in a south Indian hospital: their severity and cost involved. Pharmacoepidemiol Drug Saf 2003; 12:687-92.

12. Dormann H, Neubert A, Criegee-Rieck M, Egger T, Radespiel-Tröger M, Azaz-Livshits T, et al. Readmissions and adverse drug reactions in internal medicine: the economic impact. J Intern Med 2004; 255:653-63.

13. Forster AJ, Halil RB, Tierney MG. Pharmacist surveillance of adverse drug events. Am J Health Syst Pharm 2004; 61:1466-72.

14. Corral-Baena S, Guerrero-Aznar MD, Beltrán-García M, Salas-Turrens J. Utilización del CMBD como herramienta para la detección de acontecimientos adversos a medicamentos. Farm Hosp 2004; 28:258-65.

15. Weingart SN, Toth M, Eneman J, Aronson MD, Sands DZ, Ship AN, et al. Lessons from a patient partnership intervention to prevent adverse drug events. Int J Qual Health Care 2004; 16:499-507.
16. Nebeker JR, Hoffman JM, Weir CR, Bennett CL, Hurdle JF. High rates of adverse drug events in a highly computerized hospital. Arch Intern Med 2005; 165:1111-6.

17. Al-Tajir GK, Kelly WN. Epidemiology, comparative methods of detection, and preventability of adverse drug events. Ann Pharmacother 2005; 39:1169-74.

18. Cohen MM, Kimmel NL, Benage MK, Cox MJ, Sanders N, Spence D, et al. Medication safety program reduces adverse drug events in a community hospital. Qual Saf Health Care 2005; 14:169-74.

19. Mycyk MB, McDaniel MR, Fotis MA, Regalado J. Hospitalwide adverse drug events before and after limiting weekly work hours of medical residents to 80. Am J Health Syst Pharm 2005; 62:1592-5.

20. Camargo AL, Cardoso-Ferreira MB, Heineck I. Adverse drug reactions: a cohort study in internal medicine units at a university hospital. Eur J Clin Pharmacol 2006; 62:143-9.

21. Otero-Lopez MJ, Alonso-Hernandez P, MaderueloFernandez JA, Garrido-Corro B, Dominguez-Gil A, Sanchez-Rodriguez A. Acontecimientos adversos prevenibles causados por medicamentos en pacientes hospitalizados. Med Clin (Barc) 2006; 126:81-7.

22. Kilbridge PM, Campbell UC, Cozart HB, Mojarrad MG. Automated surveillance for adverse drug events at a community hospital and an academic medical center. J Am Med Inform Assoc 2006; 13:372-7.

23. Bond CA, Raehl CL. Clinical pharmacy services, pharmacy staffing, and adverse drug reactions in United States hospitals. Pharmacotherapy 2006; 26:735-47.

24. Hougland P, Xu W, Pickard S, Masheter C, Williams SD. Performance of International Classification of Diseases, 9th revision, clinical modification codes as an adverse drug event surveillance system. Med Care 2006; 44:629-36.

25. Lugardon S, Desboeuf K, Fernet P, Montastruc JL, Lapeyre-Mestre M. Using a capture-recapture method to assess the frequency of adverse drug reactions in a French university hospital. Br J Clin Pharmacol 2006; 62:225-31.

26. Davies EC, Green CF, Mottram DR, Pirmohamed M. Adverse drug reactions in hospital in-patients: a pilot study. J Clin Pharm Ther 2006; 31:335-41.

27. Tribino G, Maldonado C, Segura O, Diaz J. Costos directos y aspectos clínicos de las reacciones adversas a medicamentos en pacientes hospitalizados en el servicio de medicina interna de una institución de tercer nivel de Bogotá. Biomedica 2006; 26:31-41.

28. Schade CP, Hannah K, Ruddick P, Starling C, Brehm J. Improving self-reporting of adverse drug events in a West Virginia hospital. Am J Med Qual 2006; 21:335-41.

29. Rozenfeld S. Agravos provocados por medicamentos em hospitais do Estado do Rio de Janeiro, Brasil. Rev Saúde Pública 2007; 41:108-15. 
30. Sanchez-Muñoz LA, Castiella-Herrero J, SanjuánPortugal FJ, Naya-Manchado J, Alfaro-Alfaro MJ. Utilidad del CMBD para la detección de acontecimientos adversos por medicamentos. An Med Interna 2007; 24:113-9.

31. Hwang SH, Lee S, Koo HK, Kim Y. Evaluation of a computer-based adverse-drug-event monitor. Am J Health Syst Pharm 2008; 65:2265-72.

32. Pourseyed S, Fattahi F, Pourpak Z, Gholami K, Shariatpanahi SS, Moin A, et al. Adverse drug reactions in patients in an Iranian department of internal medicine. Pharmacoepidemiol Drug Saf 2009; 18:104-10.

33. Davies EC, Green CF, Taylor S, Williamson PR, Mottram DR, Pirmohamed M. Adverse drug reactions in hospital in-patients: a prospective analysis of 3695 patient-episodes. PLoS One 2009; 4:e4439.

34. Lagnaoui R, Moore N, Fach J, Longy-Boursier M, Bégaud B. Adverse drug reactions in a department of systemic diseases-oriented internal medicine: prevalence, incidence, direct costs and avoidability. Eur J Clin Pharmacol 2000; 56:181-6.

35. Rissato MA, Romano-Lieber NS, Lieber RR. Terminologia de incidentes com medicamentos no contexto hospitalar. Cad Saúde Pública 2008; 24 1965-75.

36. Bates DW, Cullen DJ, Laird N, Petersen LA, Small $\mathrm{SD}$, Servi D, et al. Incidence of adverse drug events and potential adverse drug events: implications for prevention. JAMA 1995; 274:29-34.
37. Resar RK, Rozich JD, Classen D. Methodology and rationale for the measurement of harm with trigger tools. Qual Saf Health Care 2003; 12 Suppl II: ii39-45.

38. Gomes MJVM, Reis AMM. Ciências farmacêuticas Uma abordagem em farmácia hospitalar. São Paulo: Editora Atheneu; 2001.

39. Thürmann PA. Methods and systems to detect adverse drug reactions in hospitals. Drug Saf 2001; 24:961-8.

40. Carbonin P, Pahor M, Bernabei R. Is age an independent risk factor of adverse drug reactions in hospitalized medical patients? J Am Geriatr Soc 1991; 39:1093-9.

41. van den Bemt PM, Egberts AC, Lenderink AW, Verzijl JM, Simons KA, van der Pol WS, et al. Risk factors for the development of adverse drug events in hospitalized patients. Pharm World Sci 2000; 22:62-6.

42. Bégaud B, Martin K, Fourrier A, Haramburu F. Does age increase the risk of adverse drug reactions? $\mathrm{Br} J$ Clin Pharmacol 2002; 54:550-2.

43. Evans RS, Lloyd JF, Stoddard GJ, Nebeker JR, Samore MH. Risk factors for adverse drug events: a 10-year analysis. Ann Pharmacother 2005; 39:1161-8

44. van Ryn M, Burke J. The effect of patient race and socio-economic status on physicians' perceptions of patients. Soc Sci Med 2000; 50:813-28.

45. McDowell SE, Coleman JJ, Ferner RE. Systematic review and meta-analysis of ethnic differences in risks of adverse reactions to drugs used in cardiovascular medicine. BMJ 2006; 332:1177-81.

Submitted on 12/May/2009

Final version resubmitted on 18/Sep/2009

Approved on 23/Sep/2009 